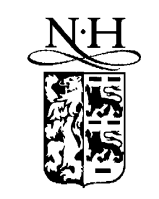

ELSEVIER

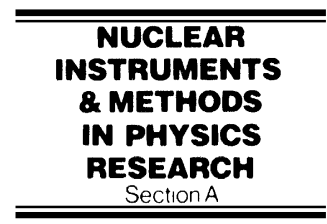

www.elsevier.com/locate/nima

\title{
Calculation of the output charge cloud from a microchannel plate
}

\author{
G.J. Price, G.W. Fraser* \\ X-ray Astronomy Grp. Space Research Centre, Department of Physics and Astronomy, University of Leicester, \\ University Road, Leicester LE1 7RH, UK \\ Received 19 February 2001
}

\begin{abstract}
The imaging performance of microchannel plate (MCP) detectors of photons and charged particles depends on the angular and energy distributions of the electrons in the output charge cloud. Measurements of these distributions are sparse and do not cover the full range of MCP operating parameters (input current, bias voltage, electrode design, channel length and channel diameter).

Our two dimensional Monte Carlo model of the avalanche process in a single microchannel yields the output energy distribution of electrons (EDOE) and angular distribution of electrons (ADOE) for arbitrary channel geometries. The model has been validated by comparison with the available experimental data and used to examine the (a) effects of current saturation and (b) novel output electrode geometries. (C) 2001 Elsevier Science B.V. All rights reserved.
\end{abstract}

PACS: $02.50 . \mathrm{Ng} ; 02.70 . \mathrm{Uu}$

Keywords: Monte Carlo model; Electron avalanche; Microchannel plate

\section{Introduction}

The spatial resolution and image linearity of microchannel plate (MCP) imaging detectors using 'progressive geometry' position encoders depend on the energy and angular distributions of the electrons in the output charge cloud. The charge 'footprint' on the readout element must be matched to the repeat pattern of the encoder in order to avoid distortion. Energetic electrons in the charge cloud may also reduce image quality by

\footnotetext{
*Corresponding author.

E-mail address: gwf@star.le.ac.uk (G.W. Fraser).
}

enlarging and distorting the footprint through secondary electron emission from the anode [1-3].

MCP electron energy distributions may be measured by varying the retarding electric field between a collector plate and the MCP output surface and recording the MCP output current [4-7]. Angular distributions may be obtained by moving a small $\left(\sim 1 \times 10^{-4}\right.$ sr $)$ detector-analyser through a range of solid angles around the output of the active channel $[8,9]$. The available experimental data, however, is sparse. In summary:

(i) For a fixed channel geometry (pore diameter $D$, channel length $L$ ) Bronshteyn et al. [9] report that the width of the ADOE increases 
with MCP bias voltage $V_{\mathrm{o}}$; Tyutikov and Tsoi [8] find the contrary dependence.

(ii) The width of the ADOE decreases slowly with the depth of the nickel-based electrode penetration ('endspoiling') into the channel from the exit face.

(iii) The high energy tail of the EDOE is suppressed by operation in current saturation mode where the signal current $I_{\mathrm{c}}$ is a significant fraction of $I_{\mathrm{s}}$, the standing current in the plate.

We now describe a Monte Carlo model of the electron avalanche in a single two dimensional microchannel which we have used to systematically investigate the dependence of the ADOE and EDOE on $D, L / D, V_{\mathrm{o}}, I_{\mathrm{c}} / I_{\mathrm{s}}$ and $h$, the extent of electrode endspoiling.

Regarding previous simulations, the early results of Guest [10] give a good approximation to EDOE data, but make no angular predictions. More recently Antonov et al. [11], Suzuki and Konno [12] and Choi and Kim [13] have reported Monte Carlo avalanche simulations. Choi and Kim do not report any EDOE or ADOE data; Suzuki and Konno describe ADOE measurements for a parallel plate electron multiplier, while Antonov et al. report only qualitative ADOE calculations with no EDOE data. The many analytical studies of MCP gain [14-19] yield no information on ADOE or EDOE.

\section{Model}

The model of the working channel has two elements: the representation of the electron emission process from the channel wall (Sections 2.1-2.3) and the calculation of the channel electric field (Section 2.4) in which electron trajectories are tracked (Section 2.5).

\subsection{Secondary electron yield $\delta$}

A microchannel plate electron multiplier is based on secondary electron emission (SEE). A charged particle or energetic photon incident on a channel wall near the channel entrance will excite

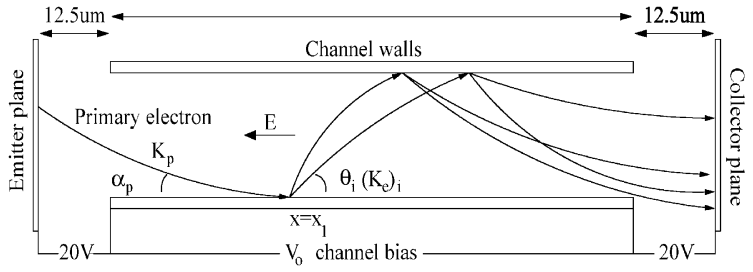

Fig. 1. Signal amplification in a microchannel. $K_{\mathrm{p}}$ and $\alpha_{\mathrm{p}}$ are the energy and grazing angle for the incident primary electron initiating the avalanche. $\theta_{i}(i=1,2 \ldots$ in the schematic shown here) and $\left(K_{\mathrm{e}}\right)_{i}$ are the emission angles and energies of the emitted secondaries, $\alpha_{i}$ and $\left(K_{\mathrm{c}}\right)_{i}$ are the corresponding collision angles and energies.

secondary electrons into vacuum. A bias applied along the length of the channel then accelerates these secondary electrons down the channel where they themselves will strike the wall. Provided the electron collision energy $K_{\mathrm{c}}$ exceeds $e V_{1}$ where $V_{1}(\sim 30 \mathrm{~V})$ is the first cross over potential, an avalanche propagates, as indicated in Fig. 1 .

According to the two dimensional channel model of Adams and Manley [14]

$K_{\mathrm{c}}=\frac{V_{\mathrm{o}}^{2} e}{4 K_{\mathrm{e}} \sin ^{2} \theta} \frac{D^{2}}{L^{2}}$

where $K_{\mathrm{e}}$ is the secondary electron emission energy and $\theta$ is the angle of emission of the electron with respect to the channel surface. $L / D$ is the channel length to diameter ratio. To zeroth order, $K_{\mathrm{c}}$ is a measure of the maximum electron energy in the EDOE. Assuming electron emission normal to the wall, $K_{\mathrm{c}}$ and $\alpha$ are constants, and the channel gain $G$ is given by

$G=\delta\left(K_{\mathrm{c}}, \alpha\right)^{\frac{e V_{\mathrm{o}}}{K_{\mathrm{c}}}}$

where $\delta$ is the secondary electron yield coefficient of the surface - the mean number of secondary electrons emitted per incident primary.

The semi-empirical models of Burke [20], Dekker [21] and Hill [22] suggest that the yield for normal incidence primaries is

$\delta\left(K_{\mathrm{c}}, \frac{\pi}{2}\right)=P_{\mathrm{s}}(0) \frac{K_{\mathrm{c}} L_{\mathrm{s}}}{\varepsilon R_{\mathrm{p}}}\left(1-\exp \left(\frac{-R_{\mathrm{p}}}{L_{\mathrm{s}}}\right)\right)$

where $P_{\mathrm{s}}(0)$ is the surface escape probability, $\varepsilon$ is the energy to create a secondary electron in the material, $L_{\mathrm{s}}$ is the secondary electron escape length and $R_{\mathrm{p}}$ is the range of the primary in the glass. One 


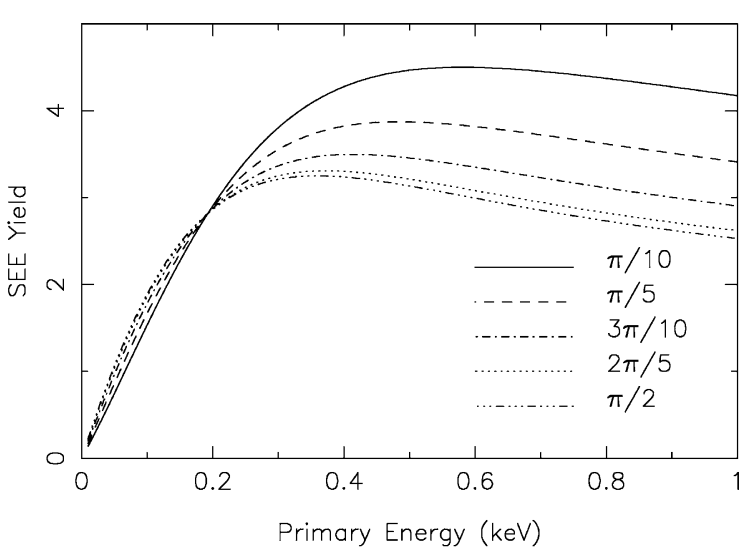

Fig. 2. SEE yield as a function of primary electron energy for five different primary grazing angles of incidence $\alpha$.

of us [23] has previously derived values for the material constants of lead silicate glass by comparison with the measurements of Refs. [22,24]; $P_{\mathrm{s}}(0)=0.15, \quad L_{\mathrm{S}}=33 \AA, \varepsilon=10 \mathrm{eV}$. For nickel, representing the nichrome or inconel end-spoiling electrodes, an analysis of the ion-induced secondary electron yield data of Von Gemmingen [25] gives; $P_{\mathrm{s}}(0)=0.024, L_{\mathrm{s}}=20 \AA, \varepsilon=6.65 \mathrm{eV}$.

The increase of $\delta$ with decreasing grazing angle of incidence $\alpha$ comes from a reduction in the distance below the surface that the secondaries are created. Guest [10] and Boucharde and Carette [26] suggest that

$$
\delta\left(K_{\mathrm{c}}, \alpha\right)=\delta\left(K_{\mathrm{c}}, \frac{\pi}{2}\right) \exp \left[p\left(K_{\mathrm{c}}\right)(1-\sin \alpha)\right]
$$

where $\quad p\left(K_{\mathrm{c}}\right)=0.7664-1.533 \exp \left(-3.598 K_{\mathrm{c}}\right)$, with $K_{\mathrm{c}}$ in $\mathrm{keV}$. Fig. 2 shows the resulting secondary electron yield function for MCP glass.

A fraction $R$ of the incident primaries are elastically scattered back into the vacuum. The lead glass data of Goff and Hendee [27] indicate that $R \ll \delta\left(R=0.17\right.$ for $\alpha=20^{\circ}$ and a primary energy of $51 \mathrm{eV} ; R=0.05$ for $\alpha=90^{\circ}$ and an energy of $300 \mathrm{eV}$ ) and so backscattering is neglected in the calculations below.

\subsection{Electron emission energies}

The energy distribution of the secondaries produced by primary electron bombardment of lead silicate glass has been thoroughly investigated

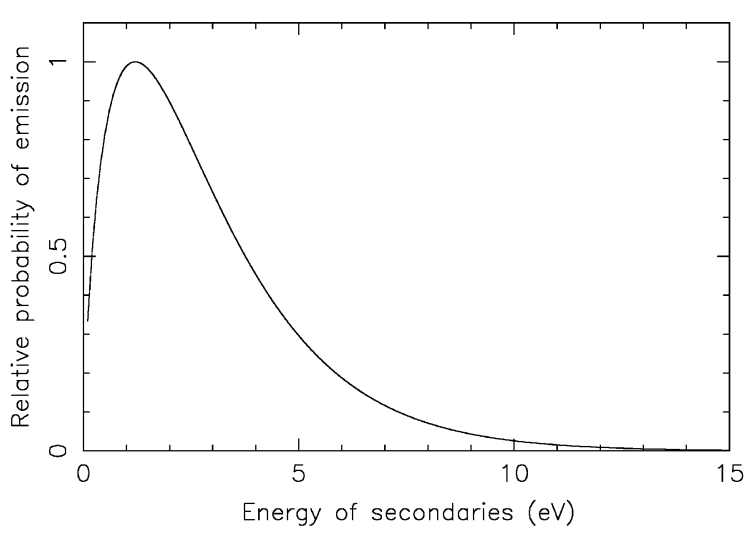

Fig. 3. Energy distribution of secondary electron emission for lead glass.

experimentally $[22,24]$. The relative probability of electron emission at an energy $K_{\mathrm{e}}$ can be expressed by the empirical formula [28].

$f\left(K_{\mathrm{e}}\right)=C\left(\frac{K_{\mathrm{e}}}{K_{\mathrm{av}}}\right)^{\beta} \exp \left(\frac{-\gamma K_{\mathrm{e}}}{K_{\mathrm{av}}}\right)$

where $C$ is a normalisation factor, $K_{\mathrm{av}}$ is the average secondary energy at emission, and $\beta$ and $\gamma$ are fit parameters. From comparisons with the data of Ref. [22] for lead silicate glass, the fit parameters are $\beta=0.7$ and $\gamma=7$. The distribution used in the calculations below is shown in Fig. 3 .

\subsection{Electron emission angles}

The angular distribution of secondary electrons produced by grazing incidence electron impact on amorphous solids is not well known. The distribution is generally assumed to be of an azimuth independent cosine from $[12,29]$ where, if $\phi$ is the angle of emission (to the normal of the surface) then the probability of emission is:

$P(\phi)=\cos \phi$.

A physical basis for this distribution is the large number of collisions undergone by secondary electrons during transport from their generation site to the surface. This hypothesis is supported by Monte Carlo simulations of the SEE process [30,31].

Some experimental evidence points to a deviation from the distribution of Eq. (6) as the grazing 
angle of the primary electrons decreases. Goff and Hendee [27] state that the greatest probability of secondary electron emission is back towards the direction of the incident electron beam. This backskewing is attributed to an increase in the probability of inelastic backscattering. There is, however, no available data on the energy dependence of this back-skewing so that in the simulations described below, the secondary electron emission distribution is unchanged from Eq. (6).

The electron energy and angle emission distributions of the nickel electrodes are assumed to be the same as those of the channel glass.

\subsection{Electric field calculation}

The channel electrostatic potential distribution $V(x, y)$ is solved, in the presence of electrode endspoiling and other perturbations, by finite difference methods. The two dimensional potential matrix is first seeded with a potential linearly increasing in $x$ and then Laplace's equation $\left(\nabla^{2} V=0\right)$ is solved by four-point Newtonian relaxation, as reported in Ref. [32]. Iteration continues until either the tolerance of $1 \times 10^{-2} \mathrm{~V}$ is reached or the number of iterations exceeds a preset value (the multiple of the $x$ and $y$ dimensions of the potential matrix).

The array elements are separated by $0.25 \mu \mathrm{m}$ in $x$ and $y$ so that in a typical geometry, the potential is stored in a $5000 \times 80$ element array. Using a $433 \mathrm{MHz}$ Dec Alpha workstation, a typical potential generation takes $\sim 8 \mathrm{~h}$. An example is shown in Fig. 4.

\subsection{Electron transport}

Each avalanche is initiated by a single primary electron of random energy $K_{\mathrm{p}}$ in the range $100-$ $600 \mathrm{eV}$ and incident angle $\alpha_{\mathrm{p}}$ randomly selected within the channel entrance cone. The model assumes a $20 \mathrm{~V}$ electron accelerating potential between the emitter plane and channel entrance plane (and between the exit and collector planes). It has been confirmed that the calculated EDOE and ADOE are independent of the starting conditions.

The grazing angle of the primary is calculated at the position of first strike $\left(x=x_{1}\right.$ in Fig. 1) and a

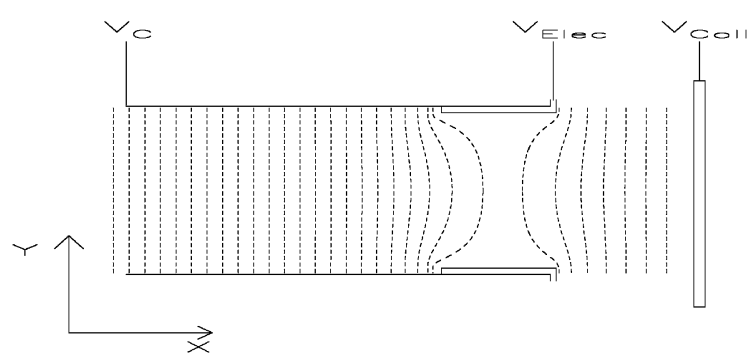

Fig. 4. Equipotentials near the exit of an end-spoiled channel. $V_{\mathrm{c}}=-75 \mathrm{~V}$; electrode potential $V_{\text {Elec }}=-20 \mathrm{~V}$; collector potential $V_{\text {coll }}=0 \mathrm{~V}$.

value of $\delta$ calculated from Eqs. (3) and (4). The number of emitted secondaries is then chosen randomly from a Poissonian distribution with mean $\delta$. For each secondary, an emission angle and energy are assigned from the distributions described by Eqs. (5) and (6), respectively. The individual electrons are traced in the potential distribution $V(x, y)$ until they next strike the channel wall (lead silicate glass or nickel electrode) or they exit the channel. The equations of motion are solved at $0.1 \mathrm{ps}$ intervals (the transit time of an electron avalanche in a microchannel is typically $100 \mathrm{ps)}$ using the formulae of Klemperer and Barnett [33]. The calculated ADOE and EDOE typically contain $\sim 20$ electrons.

\section{Comparison of model with experiment}

\subsection{Linear operation}

Figs. 5 and 6 compare experimental EDOE [6] and ADOE [8] data together with our calculations for channels of the same parameters. The calculated EDOE shows very good agreement at low energy, the fit deviating at higher energy. The calculated ADOE has the same width and shape as the measured distribution, but is shifted by $-1.0^{\circ}$ with respect to the results of Tyutikov and Tsoi. This offset, an artefact of the model being two dimensional, is removed from Fig. 6 so that the otherwise excellent fit can be seen. Despite this two dimensional nature of the Monte Carlo model and the consequent under-estimation of the number of 


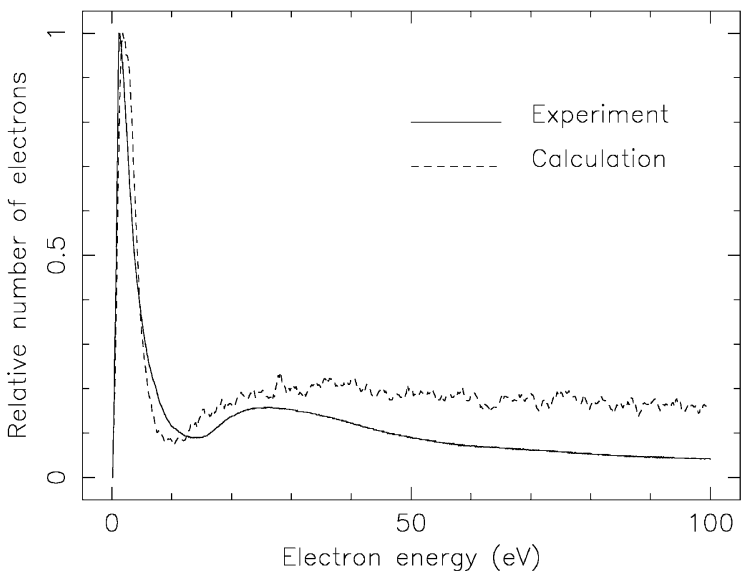

Fig. 5. Calculated and measured [6] EDOEs. $D=12.5 \mu \mathrm{m}$; $L / D=40: 1 ; V_{\mathrm{o}}=1000 \mathrm{~V} ; h / D=0.9$. The $20 \mathrm{~V}$ offset in calculated data due to the collecting field has been removed in this figure. The maximum emission energy indicated by Eq. (1) is $156 \mathrm{eV}$ for $K_{\mathrm{e}}=1 \mathrm{eV}$ and $\theta=\pi / 2$.

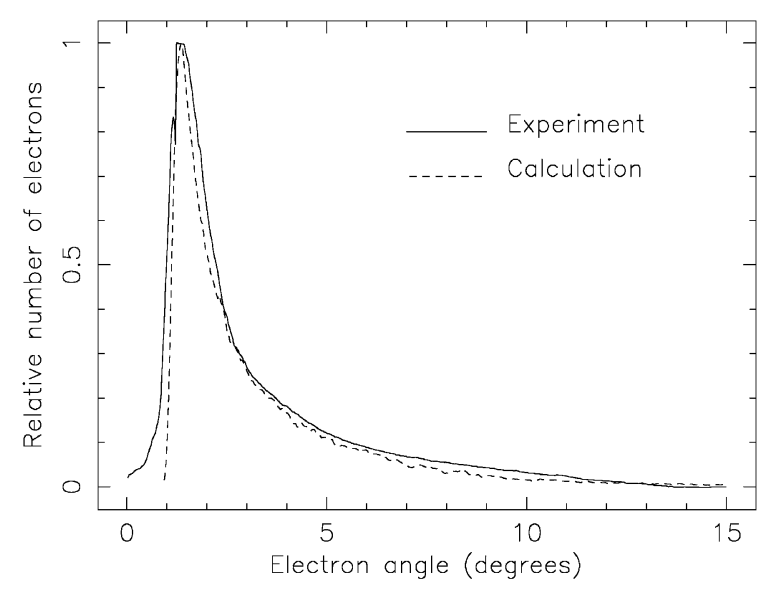

Fig. 6. Calculated and measured [8] ADOEs. $D=20 \mu \mathrm{m}$; $L / D=60: 1 ; V_{\mathrm{o}}=1000 \mathrm{~V} ; h / D=1.0$. A $-1.0^{\circ}$ offset of the calculated distribution with respect to that of the measured one, an artefact of the two dimensional nature of the model, is removed in this figure.

collisions at small $\alpha /$ high $\delta$, we do reproduce the salient features of the measured distributions.

Further calculations in 'simple' channel geometries show that increasing the channel electric field strength (decreasing the aspect ratio $L / D$ or increasing the applied bias $V_{\mathrm{o}}$ ) increases the number of low energy divergent electrons produced by a channel and vice-versa. In other words,
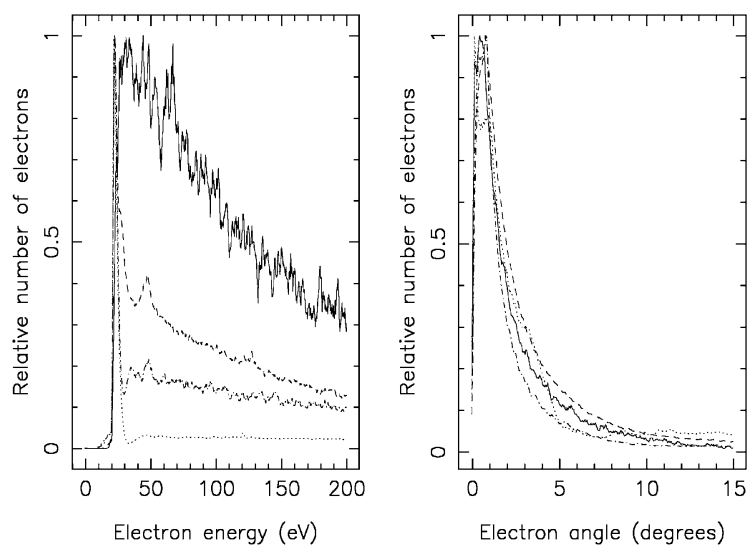

Fig. 7. EDOEs (left) and ADOEs (right) for a $12 \mu \mathrm{m}, 40: 1$ channel at $800 \mathrm{~V}$ with various degrees of end-spoiling. The solid, dashed, dash-dot and dotted lines correspond to $h / D=0$, $0.5,1$ and 3 , respectively. The $20 \mathrm{~V}$ offset corresponds to the collector field.

the trend for ADOE to broaden with increased $V_{\mathrm{o}}$ [9] is confirmed.

According to Bronshteyn et al. [9], the halfwidth of the ADOE peak decreases slowly with the degree of end-spoiling $h / D$. The angular halfwidths measured by these authors lie in the range $5-15^{\circ}$. Fig. 7 shows the calculated EDOEs/ADOEs which result from the variation of $h / D$ in a standard channel geometry. Our ADOEs are narrower than those of Ref. [9], and show no systematic variation with $h / D$. Increasing the degree and end-spoiling in the model has, however, a marked effect on the EDOE, progressively suppressing the tail of the distribution until, for $h / D=3$, the electron energy distribution consists mainly of a sub- $10 \mathrm{eV}$ spike. This suppression of high energy electrons is observed experimentally by Koshida [34] for $h / D=1,1.5$ and 2.0. Koshida speculated and our model confirms that the electrons in the EDOE peak originate in the constant potential region at the channel exit. Changing $P_{\mathrm{s}}(0)$ for the electrode material, for example, directly influences the relative weights of the EDOE spike and tail.

\subsection{Operation in current saturation}

In the previous section we assumed that the input current and channel gain are both low, so 
that the electric field in the channel is determined only by the applied voltage and the electrode penetration. For high gains, positive wall charging [23], due to the failure of the channel to replace charge emitted from the wall during the avalanche, acts to reduce the electric field strength at the channel exit.

When the output current $I_{\mathrm{c}}$ is a significant fraction of the standing current, $I_{\mathrm{S}}=V_{\mathrm{o}} / R_{\mathrm{MCP}}$, space charge effects also diminish the electric field. Here, $R_{\mathrm{MCP}}$ is the resistance of a microchannel plate. We have extended our ADOE/EDOE model to deal with current saturation, where the formulae of Harris [15] can be used to estimate the variation of the electric field along the channel axis as $I_{\mathrm{c}} / I_{\mathrm{s}}$ is increased.

It is not realistic to exactly model such a process, since the channel potential would have to be recalculated after each secondary electron emission. Instead, we estimate the steady state potential in the channel and then trace the trajectory of a single electron in the field of many others.

Harris [15] has derived an equation that allows the calculation of the required channel potential. By considering the total current in a channel (wall current plus space current) as a conserved quantity and considering changes in wall conductivity as the avalanche progresses; it can be shown that

$$
\frac{\mathrm{d} E_{\mathrm{R}}}{\mathrm{d} x}=-\frac{4 K_{\mathrm{e}}}{E_{\mathrm{o}} E_{\mathrm{R}} D^{2}} \frac{\left(1-E_{\mathrm{R}}\right)(\delta-1)}{\left(1+2 A e E_{\mathrm{o}}^{2} E_{\mathrm{R}} \frac{\left(1-E_{\mathrm{R}}\right)}{\left(1+A e E_{\mathrm{o}}^{2} E_{\mathrm{R}}^{2}\right)}\right)}
$$

where $E_{\mathrm{R}}$ is the relative field strength, $E_{\mathrm{o}}$ is the field strength at the entrance of the channel, $\delta$ is the secondary electron yield and $A=1 / W$, where $W$ is the electron-hole pair creation energy (3.65 eV for $\mathrm{Si}$ [35]). The space current to standing current ratio at a depth $x$ in the channel is

$\frac{I_{\mathrm{c}}(x)}{I_{\mathrm{s}}}=\frac{1-E_{\mathrm{R}}(x)}{1+A e E_{\mathrm{o}}^{2} E_{\mathrm{R}}^{2}(x)}$.

The actual wall current only flows near the surface of the channel where the lead glass has been reduced to a semi-conducting state. Bombardment of this layer of reduced glass with electrons will
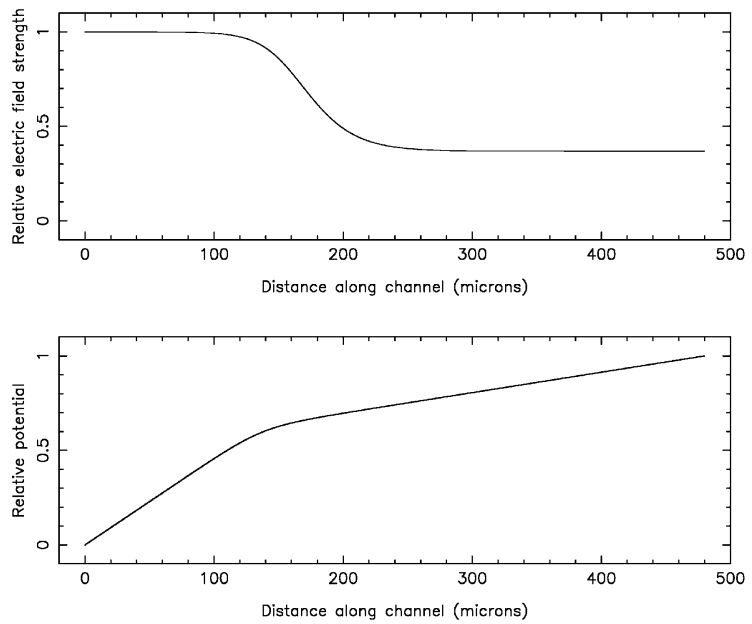

Fig. 8. Relative potential (bottom) and electric field strength (top) as a function of distance along a $12 \mu \mathrm{m}, 40: 1$ channel biased with $800 \mathrm{~V}$ and with $0.9 \mathrm{D}$ endspoiling. Calculated from Eq. (7). $I_{\mathrm{c}} / I_{\mathrm{s}} \sim 0.6$ in this example.

increase its conductivity via the creation of mobile electrons (secondary electrons that have not yet migrated to the surface and been emitted) and the corresponding holes. Fig. 8 shows the potential and electric field strength as functions of the distance along a uniform channel calculated using Eq. (7). $I_{\mathrm{c}} / I_{\mathrm{s}} \sim 0.6$ in this example.

This potential function was then used as the initial trial solution $V(x, y)$ for calibration of the channel field in the presence of non-zero endspoiling.

Experimentally, Koshida et al. [6] have found that as $I_{\mathrm{c}} / I_{\mathrm{s}}$ increases, the fraction of electrons with output energies in excess of $50 \mathrm{eV}$ decreases. Fig. 9 compares in detail the measured [6] and calculated EDOEs for a linear (non-saturated) and saturated microchannel. The agreement between the two is excellent.

\section{Applications of the model}

The model, although only two dimensional, produces good agreement with the available experimental data and can thus be used to predict 

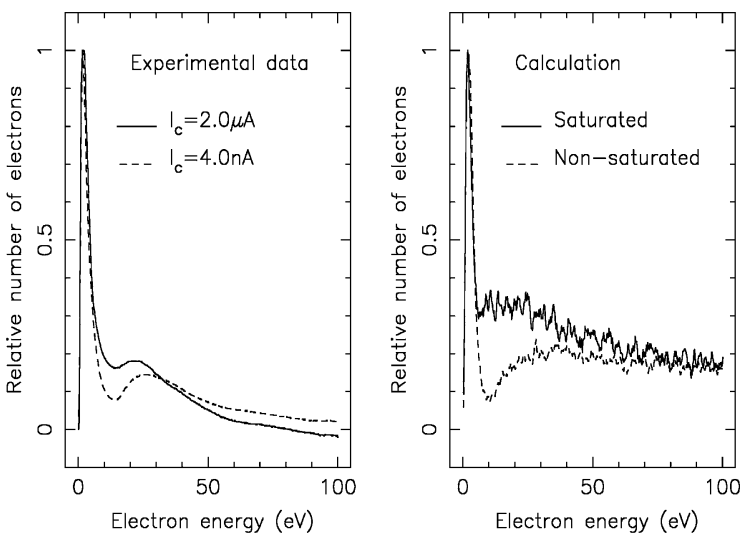

Fig. 9. Measured [6] (left) and calculated (right) changes in EDOE with degree of current saturation. $D=12 \mu \mathrm{m}$; $L / D=40: 1 ; V_{\mathrm{o}}=1000 \mathrm{~V}$ and $h / D=0.9$.

the EDOE and ADOE of unknown channel geometries with some degree of confidence. Examples of interest include:

(i) the influence of alkali halide coatings on the channel walls [6];

(ii) etched silicon MCPs [36],

provided the secondary electron yield parameters of the channel walls are known.

Proposed channel modifications can be appraised before costly experimental runs. The model also allows encoders to be matched to MCPs with an appropriate output charge cloud for maximum resolution.

A third example, the addition of an electron lens to the microchannel output, is discussed below.

\subsection{Electron lens}

The addition of micro-fabricated electrostatic lenses to the output of each channel on a microchannel plate has been suggested as a means of narrowing the ADOE [37]. The lens structure is shown schematically in Fig. 10.

Fig. 11 shows the potential distribution in such a lens system. Electrons initially parallel to the channel axis have been traced through the potential and weak focusing is observed. In this

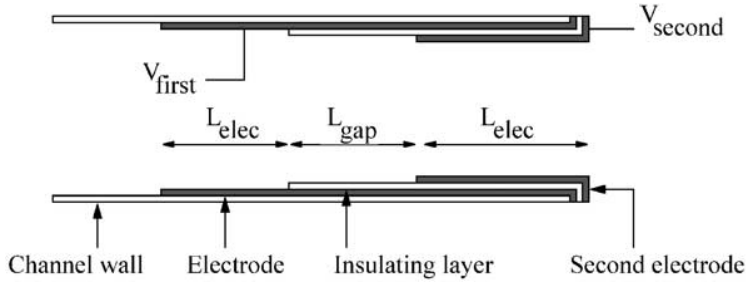

Fig. 10. Channel exit lens [37]. A conducting electrode is evaporated onto the channel in the normal way to a depth of $2 L_{\text {elec }}+L_{\text {gap }}$. An $\mathrm{SiO}_{2}$ insulating over-layer of length $L_{\text {elec }}+$ $L_{\text {gap }}$ is then deposited, and then finally a second electrode of length $L_{\text {elec }}$. The applied potentials are $V_{\text {first }}$ and $V_{\text {second }}$.

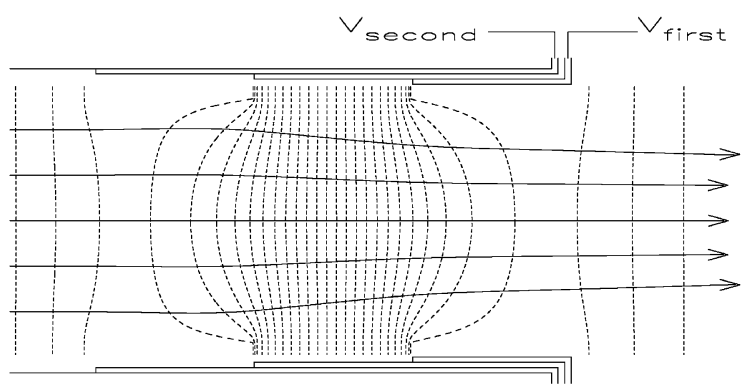

Fig. 11. Equipotential map of an electrostatic lens mounted at a microchannel exit. The trajectories of electrons with velocities initially parallel to the channel axis are shown. Contours are $5.5 \mathrm{~V}$ apart; $D=12 \mu \mathrm{m} ; L / D=40: 1 ; L_{\mathrm{elec}}=12 \mu \mathrm{m} ; L_{\mathrm{gap}}=12 \mu \mathrm{m}$; $V_{\text {first }}=-20 \mathrm{~V}$ and $V_{\text {second }}=-170 \mathrm{~V}$.

calculation the first and second electrodes were separated by $150 \mathrm{~V}$; it is not clear if it is possible to implement the electrode structure with such a large voltage over the insulating structure. Raytraces run with lower potential differences indicate a lack of focusing; for electrode potential differences of $70 \mathrm{~V}$ or less, mild dispersion is observed.

\subsection{Results}

The EDOE and ADOE resulting from calculations with the lens system in place are shown in Fig. 12. The ADOEs show there is, unfortunately no discernible focusing effect from the lenses. The EDOEs exhibit a suppressed intermediate-energy region relating to the electrode gap, with electron 

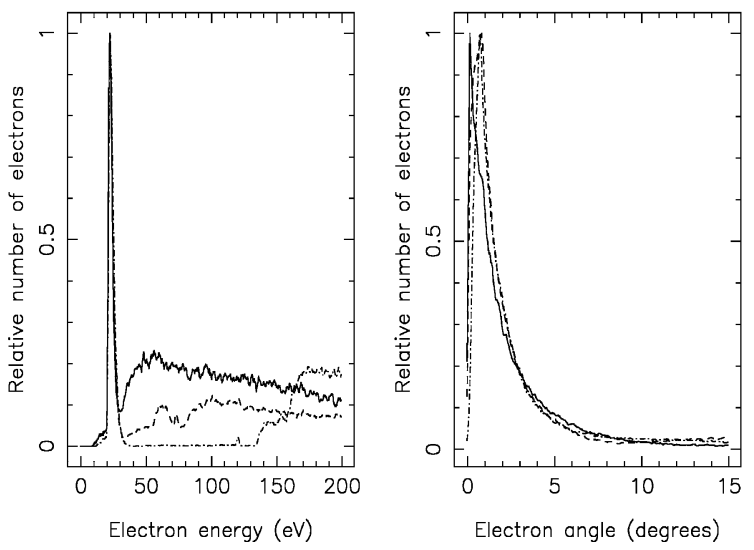

Fig. 12. EDOEs (left) and ADOEs (right) for a $12 \mu \mathrm{m}, 40: 1$ channel with and without an electron lens. The solid line represents a normal channel $\left(h / D=0.9, \quad V_{\mathrm{o}}=1000 \mathrm{~V}\right)$. The dashed and dash-dot lines correspond to channels with an electron lens $\left(L_{\text {elec }}=12 \mu \mathrm{m} ; L_{\text {gap }}=12 \mu \mathrm{m} ; V_{\text {first }}=-20 \mathrm{~V}\right.$ with $V_{\text {second }}=-90 \mathrm{~V}$ and $-170 \mathrm{~V}$, respectively).

emission seen from the constant potential regions of both the lens electrodes.

\section{Conclusions}

We have developed a two dimensional model of a single microchannel amplifier, including current saturation mode. Energy and angular distributions of electrons in the output charge cloud have been calculated which agree well with experimental evidence.

We have confirmed the important influence of electrode endspoiling on the EDOE and ADOE of a microchannel. Otherwise the distributions are rather robust; that is, largely independent of channel parameters.

\section{Acknowledgements}

This work was supported in part by a US government SBIR Grant from BMDO/DARPA through NOVA Scientific, Inc., Sturbridge, MA, USA. The authors wish to thank Bruce Feller (NOVA Scientific) and Harry Lockwood (Lockwood Group, Newton, MA) for many helpful discussions. GJP acknowledges the receipt of a PPARC Ph.D. studentship.

\section{References}

[1] M.L. Edgar, R. Kessel, J.S. Lapington, D.M. Walton, Rev. Sci. Instrum. 60 (1989) 3673.

[2] J.S. Lapington, Nucl. Instr. and Meth. A 392 (1997) 336.

[3] J.S. Lapington, L.B.C. Worth, Proc. SPIE. 3445 (1998) 546.

[4] N. Koshida, S. Yoshida, Rev. Sci. Instrum. 50 (1979) 177.

[5] N. Koshida, M. Kunii, S. Yoshida. Rev. Sci. Instrum. 51 (1980) 365

[6] N. Koshida, M. Midorikawa, Y. Kiuchi, Adv. Electron. Electron Phys. B 64 (1985) 337.

[7] N. Koshida, M. Hosobuchi, Rev. Sci. Instrum. 56 (1985) 1329.

[8] A.M. Tyutikov, L.B. Tsoi, Sov. J. Opt. Technol. 43 (1976) 86.

[9] I.M. Bronshteyn, A.V. Yevdokimov, A.M. Tyutikov, V.M. Stozharov, Radio Eng. Electron. Phys. 24 (1979) 150.

[10] A.J. Guest, Acta Electron. 14 (1) (1971) 79.

[11] A.V. Antonov, V.V. Apanasovich, E.G. Novikov, Proc. SPIE. 2859 (1996) 281.

[12] S. Suzuki, T. Konno, Rev. Sci. Instrum. 64 (1993) 436.

[13] Y.S. Choi, J.M. Kim, IEEE Trans. Electron. Devices 47 (2000) 1293.

[14] J. Adams, B.W. Manley, IEEE Trans. Nucl. Sci. NS-13 (1996) 100.

[15] L.A. Harris, Rev. Sci. Instrum. 42 (1971) 987.

[16] E.H. Eberhardt, Appl. Opt. 18 (1979) 1418.

[17] I.P. Csorba, Appl. Opt. 19 (1980) 3863.

[18] E.H. Eberhardt, IEEE Trans. Nucl. Sci. NS-28 (1981) 712.

[19] H.O. Funsten, D.M. Suszcynsky, R.W. Harper, Rev. Sci. Instrum. 67 (1996) 3478.

[20] E.A. Burke, IEEE Trans. Nucl. Sci. NS-24 (1977) 2505.

[21] A.J. Dekker, Solid State Physics: Secondary Electron Emission, Academic Press, New York, 1958.

[22] G.E. Hill, Adv. Electron. Electron Phys. A 40 (1976) 153.

[23] G.W. Fraser, J.F. Pearson, G.C. Smith, M. Lewis, M.A. Barstow, IEEE Trans. Nucl. Sci. NS- 30 (1983) 455.

[24] A. Authinarayanan, R.W. Dudding, Adv. Electron. Electron Phys. A 40 (1976) 167.

[25] U. Von Gemmingen, Surf. Sci. 120 (1982) 334.

[26] C. Bouchard, J.D. Carette, J. Appl. Phys. 50 (1979) 7168.

[27] R.F. Goff, C.F. Hendee, Proceedings of the 27th Annual Conference Phys. Elec., 1967.

[28] I.R. Petrovna, Yu.A. Flegontov, Sov. J. Opt. Tech. 55 (1988) 210.

[29] J. Kawarabayashi, H. Takahashi, I. Iguchi, M. Nakazawa, A. Takahashi, R. Tokue, Nucl. Instr. and Meth. A 353 (1994) 172.

[30] J. Kawata, K. Ohya, I. Mori, Jpn. J. Appl. Phys. 31 (1992) 1453. 
[31] J. Kawata, K. Ohya, Jpn. J. Appl. Phys. 36 (1997) 2325.

[32] G.W. Fraser, M.A. Barstow, J.F. Pearson, M.J. Whiteley, M. Lewis, Nucl. Instr. and Meth. A 224 (1984) 272.

[33] O. Klemperer, M.E. Barnett, Electron Optics, Cambridge University Press, Cambridge, 1971.

[34] N. Koshida, Rev. Sci. Instrum. 57 (1986) 354.
[35] G.W. Fraser, X-ray Detectors in Astronomy, Cambridge University Press, Cambridge, 1989.

[36] C.P. Beetz, R. Boerstler, J. Steinbeck, B. Lemieux, D.R. Winn, Nucl. Instr. and Meth. A 442 (2000) 443.

[37] R.F. Floryan, Focussed output microchannel plate for image intensifier tube, US Patent No. 5, 563, 653, 1996. 